Check for updates

For numbered affiliations see end of article.

Correspondence to:

Yassine Ochen yassineochen@gmail.com (ORCID 0000-0002-7187-2055) Additional material is published online only. To view please visit the journal online.

Cite this as: $B M / 2018 ; 364: k 5120$ http://dx.doi.org/10.1136/bmj.k5120

Accepted: 14 November 2018

\title{
Operative treatment versus nonoperative treatment of Achilles tendon ruptures: systematic review and meta-analysis
}

\author{
Yassine Ochen, ${ }^{12}$ Reinier B Beks, ${ }^{13}$ Mark van Heijl, ${ }^{34}$ Falco Hietbrink, ${ }^{1}$ Luke P H Leenen, ${ }^{1}$ \\ Detlef van der Velde, ${ }^{5}$ Marilyn Heng, ${ }^{2}$ Olivier van der Meijden, ${ }^{6}$ Rolf H H Groenwold, ${ }^{7}$ \\ R Marijn Houwert ${ }^{1}$
}

\section{ABSTRACT}

OBJECTIVES

To compare re-rupture rate, complication rate, and functional outcome after operative versus nonoperative treatment of Achilles tendon ruptures; to compare re-rupture rate after early and late full weight bearing; to evaluate re-rupture rate after functional rehabilitation with early range of motion; and to compare effect estimates from randomised controlled trials and observational studies.

DESIGN

Systematic review and meta-analysis.

DATA SOURCES

PubMed/Medline, Embase, CENTRAL, and CINAHL databases were last searched on 25 April 2018 for studies comparing operative versus nonoperative treatment of Achilles tendon ruptures.

\section{STUDY SELECTION CRITERIA}

Randomised controlled trials and observational studies reporting on comparison of operative versus nonoperative treatment of acute Achilles tendon ruptures.

\section{DATA EXTRACTION}

Data extraction was performed independently in pairs, by four reviewers, with the use of a predefined data extraction file. Outcomes were pooled using random effects models and presented as risk difference, risk ratio, or mean difference, with $95 \%$ confidence interval. RESULTS

29 studies were included-10 randomised controlled trials and 19 observational studies.

\section{WHAT IS ALREADY KNOWN ON THIS TOPIC}

Rupture of the Achilles tendon is a frequently encountered injury, with recent studies indicating an increase in incidence of Achilles tendon ruptures

Meta-analyses of randomised controlled trials have shown operative treatment to significantly reduce the risk of tendon re-rupture compared with nonoperative treatment (reported risk difference 5-7\%)

However, operative treatment leads to a significant increase in other complications compared with nonoperative treatment, with a reported risk difference of $16-21 \%$

\section{WHAT THIS STUDY ADDS}

Operative treatment of acute Achilles tendon ruptures reduced the risk of rerupture compared with nonoperative treatment

However, re-rupture rates were low and differences between treatment groups were small, with a risk difference of $1.6 \%$

Operative treatment resulted in a higher risk of other complications than nonoperative treatment (risk difference $3.3 \%$ ), mostly attributable to increased risk of infection
The 10 trials included 944 (6\%) patients, and the 19 observational studies included 14918 (94\%) patients. A significant reduction in re-ruptures was seen after operative treatment $(2.3 \%)$ compared with nonoperative treatment (3.9\%) (risk difference $1.6 \%$; risk ratio $0.43,95 \%$ confidence interval 0.31 to $\left.0.60 ; \mathrm{P}<0.001 ; \mathrm{I}^{2}=22 \%\right)$. Operative treatment resulted in a significantly higher complication rate than nonoperative treatment $(4.9 \% \vee 1.6 \%$; risk difference $3.3 \%$; risk ratio $2.76,1.84$ to 4.13; $\left.\mathrm{P}<0.001 ; I^{2}=45 \%\right)$. The main difference in complication rate was attributable to the incidence of infection (2.8\%) in the operative group. A similar reduction in re-rupture rate in favour of operative treatment was seen after both early and late full weight bearing. No significant difference in re-rupture rate was seen between operative and nonoperative treatment in studies that used accelerated functional rehabilitation with early range of motion (risk ratio $0.60,0.26$ to 1.37 ; $\mathrm{P}=0.23 ; \mathrm{I}^{2}=0 \%$ ). No difference in effect estimates was seen between randomised controlled trials and observational studies.

\section{CONCLUSIONS}

This meta-analysis shows that operative treatment of Achilles tendon ruptures reduces the risk of re-rupture compared with nonoperative treatment. However, re-rupture rates are low and differences between treatment groups are small (risk difference $1.6 \%$ ). Operative treatment results in a higher risk of other complications (risk difference 3.3\%). The final decision on the management of acute Achilles tendon ruptures should be based on patient specific factors and shared decision making. This review emphasises the potential benefits of adding high quality observational studies in meta-analyses for the evaluation of objective outcome measures after surgical treatment.

\section{Introduction}

Rupture of the Achilles tendon is a frequently encountered injury, with an incidence of 31 per 100000 per year, and is most common in the young to middle aged active population, with a reported mean age ranging from 37 to 44 years. ${ }^{12}$ Recent studies indicate that the incidence of Achilles tendon rupture is still increasing owing to a more active older population. ${ }^{2}$ Injury of the Achilles tendon can be debilitating because of its role in ambulation and activity, affecting both athletes and non-athletes. The management of acute Achilles tendon ruptures-operative or nonoperative treatment-is much debated. ${ }^{2}$ 
Several meta-analyses of randomised controlled trials (RCTs) have shown that operative treatment significantly reduces the risk of tendon re-rupture compared with nonoperative treatment, with a reported risk difference in re-rupture rate varying from $5 \%$ to $7 \%$. $^{3-6}$ However, operative treatment leads to a significant increase in other complications such as infection, deep vein thrombosis, and sural nerve injury, with a reported risk difference varying from $16 \%$ to $21 \%$. $^{346}$ The incidence of operative treatment has declined over the past decade as a result of multiple RCTs showing comparable results between operative and nonoperative treatment. ${ }^{12}$

A recent systematic review of overlapping metaanalyses evaluated nine meta-analyses that compared operative and nonoperative treatment of Achilles tendon ruptures. The discordance found among the nine meta-analyses indicated that further investigation is warranted as rehabilitation protocols, weight bearing restrictions, and treatment modalities have evolved. ${ }^{7}$

Systematic reviews and meta-analyses of RCTs are considered the highest level of evidence for the evaluation of treatment effects. However, several reports have shown that little evidence exists for significant differences in effect estimates between RCTs and observational studies. ${ }^{8-11}$ The addition of observational studies in meta-analyses increases sample size, which could enable the evaluation of small treatment effects and infrequent outcome measures. Furthermore, observational studies might provide insight into a variety of populations and long term effects compared with the usually highly selected patient populations in RCTs. ${ }^{12}{ }^{13}$ Both RCTs and observational studies are increasingly used in orthopaedic trauma meta-analyses for the evaluation of treatment effects. ${ }^{14-17}$

The primary aim of this systematic review and metaanalysis was to compare re-rupture rate, complication rate, and functional outcome after operative versus nonoperative treatment of acute Achilles tendon ruptures. Secondly, we sought to evaluate re-rupture rate after early and late full weight bearing and compare re-rupture rate after functional rehabilitation with early range of motion. Finally, we compared effect estimates obtained from RCTs and observational studies.

\section{Methods}

This systematic review and meta-analysis was performed and reported according to the Meta-analysis Of Observational Studies in Epidemiology (MOOSE) and the Preferred Reporting Items for Systematic Reviews and Meta-Analyses (PRISMA) checklists. ${ }^{18-20}$ A published protocol for this review does not exist.

\section{Search strategy and selection criteria}

We last searched the PubMed/Medline, Embase, CENTRAL, and CINAHL databases on 25 April 2018 for studies comparing operative versus nonoperative treatment of Achilles tendon ruptures. The search syntax is provided in supplementary table A. Duplicate articles were removed. Two reviewers (YO, RHHG) independently screened titles and abstracts for eligibility of identified studies. All published comparative studies, both RCTs and observational studies, reporting on the comparison of operative versus nonoperative treatment of acute Achilles tendon ruptures were eligible for inclusion.

After title and abstract screening, the same two reviewers (YO, RHHG) independently reviewed full text articles. Inclusion criteria were acute Achilles tendon rupture, operative treatment (open or minimally invasive surgery) versus nonoperative treatment (cast immobilisation or functional bracing), treatment within four weeks of rupture, age 16 years or older, and reporting of re-rupture rate, complication rate, or functional outcome. Exclusion criteria were delayed presentation (treatment more than four weeks after rupture), treatment for re-rupture, language other than English, no availability of full text article, and letters, meeting proceedings, and case reports. We had no inclusion restrictions based on weight bearing status or functional rehabilitation protocol. Disagreements on eligibility of full text articles were resolved by consensus or by discussion with a third reviewer (RMH). References of included studies were screened, and backwards citation tracking was performed using Web of Science to identify articles not found in the original literature search.

\section{Data extraction}

Four reviewers (YO, RHHG, RMH, RBB) extracted data independently in pairs, using a predefined data extraction file. The following baseline characteristics were extracted from the included studies: first author, year of publication, study design, country in which the study was performed, study period, number of included patients, operative method, nonoperative method, full weight bearing status, functional rehabilitation protocol, and mean follow-up. Studies reporting on patient cohorts described in previously published articles were excluded or merged.

\section{Quality assessment}

The same four reviewers (YO, RHHG, RMH, RBB), in pairs, independently assessed the methodological quality of included studies by using the Methodological Index for Non-Randomised Studies (MINORS). ${ }^{21}$ The MINORS is a validated instrument for the assessment of methodological quality and clear reporting of nonrandomised surgical studies, resulting in a score ranging from 0 to 24 for comparative studies. ${ }^{21}$ In this study the assessment of methodological quality resulted in a score ranging from 0 to 24 for RCTs and prospective cohort studies. The methodological quality of retrospective cohort studies resulted in a score ranging from 0 to 18 . The MINORS criteria for prospective collection of data, loss to followup, and prospective calculation of study size were not applicable to the retrospective cohort studies. Details on the methodological quality assessment are 
provided in supplementary table B. Disagreements were resolved by consensus.

\section{Primary and secondary outcomes}

The primary outcome measure was re-rupture rate after operative or nonoperative treatment. Secondary outcome measures included complication rate, functional outcome scores, return to sporting activity, and return to work after operative or nonoperative treatment. We defined complication rate as the rate of complications other than re-rupture. Complications included reports of wound infection, sural nerve injury, deep vein thrombosis, and pulmonary embolism. Functional outcome scores included the Achilles Tendon Rupture Score (ATRS). ${ }^{22}$ We subdivided functional outcome scores according to follow-up, into short term (one year of less) and long term (more than one year). We defined return to sporting activity as the duration in months before resumption of sports and return to work as the duration in weeks before resuming work. In studies that reported on both open and minimally invasive surgery, we used the combined outcome measures.

\section{Statistical analysis}

We present all continuous variables as mean value with standard deviation or range. We converted continuous variables to mean and standard deviation if sufficient information was available, using the methods described in the Cochrane Handbook for Systematic Reviews of Interventions. ${ }^{23}$ We extracted dichotomous variables as absolute number and percentage, pooled them using the Mantel-Haenszel method, and presented them as risk difference and risk ratio with 95\% confidence interval. We pooled continuous outcomes by using the inverse variance weighting method and presented them as mean difference with $95 \%$ confidence interval. We used random effects models for all analyses. We assessed statistical heterogeneity between studies by visual inspection of forest plots and by the $\mathrm{I}^{2}$ and $\chi^{2}$ statistics for heterogeneity. We used the overall effect $\mathrm{Z}$ test to determine the significance level for treatment effects. All analyses were stratified according to study design-RCTs or observational studies. We assessed differencein effect estimates between the two subgroups as described in the Cochrane Handbook for Systematic Reviews of Interventions. ${ }^{23}$ The significance level for difference in effect estimates across the subgroups was determined by the test for subgroup differences. We defined the significance level for treatment effects and differences across the subgroups as a $\mathrm{P}$ value below 0.05 . We assessed potential publication bias by visual inspection of funnel plots with risk ratio and standard error. $^{24}$ We used Review Manager (RevMan, version 5.3.5) for all statistical analyses. ${ }^{25}$ We further assessed publication bias with Begg's and Egger's statistical tests using Stata 13.1.

\section{Primary sensitivity analyses}

We did sensitivity analyses for the primary outcome, including studies with an early (four weeks or less) and late (more than four weeks) full weight bearing status after treatment. Studies reporting on both an early and a late full weight bearing cohort were accordingly divided for sensitivity analysis. We did an additional sensitivity analysis for the primary outcome with studies that included an accelerated functional rehabilitation protocol. We defined accelerated functional rehabilitation as the start of early range of motion within three weeks after nonoperative treatment. Rehabilitation with functional bracing systems with successive fixed degrees of plantar flexion, which did not allow for free range of motion, were not considered as accelerated rehabilitation.

\section{Secondary sensitivity analyses}

We did secondary sensitivity analyses for high quality studies and year of study period, regarding rerupture rate and complication rate. We defined high quality studies as RCTs or prospective cohort studies with a MINORS score of 16 or higher (range 0 -24) or retrospective cohort studies with a MINORS score of 12 or higher (range 0-18). We did additional sensitivity analyses with studies that included patients after the study period 2000, to account for the development of new rehabilitation protocols, operative techniques, and nonoperative treatment modalities.

\section{Patient and public involvement}

No patients were involved in setting the research question or the outcome measures, nor were they involved in developing plans for or implementation of the study. No patients were asked to advise on interpretation or writing up of results. There are no plans to disseminate the results of the research to study participants or the relevant patient community.

\section{Results \\ Search}

Figure 1 shows a flowchart of the literature search and study selection. Full text articles could not be obtained for three studies. ${ }^{26-28}$ Four studies reported on patient cohorts described in previously published articles and were excluded or merged with the original studies. ${ }^{29-32}$ This resulted in the final inclusion of 29 studies for analyses in this systematic review and metaanalysis-10 RCTs and 19 observational studies. ${ }^{33-61}$

\section{Baseline study characteristics}

The 29 studies included 15862 patients, of whom 9375 were treated operatively and 6487 nonoperatively. The overall weighted mean age was 41 (range 17-86) years, 41 years in the operative group and 44 years in the nonoperative group. Overall, the studies included 11779 (74\%) males. Overall follow-up ranged from 10 to 95 months. Table 1 shows the baseline characteristics for both RCTs and observational studies. In addition, supplementary table $\mathrm{C}$ shows the treatment characteristics of all included studies.

The 10 RCTs included 944 (6\%) patients; 469 patients were treated operatively and 475 


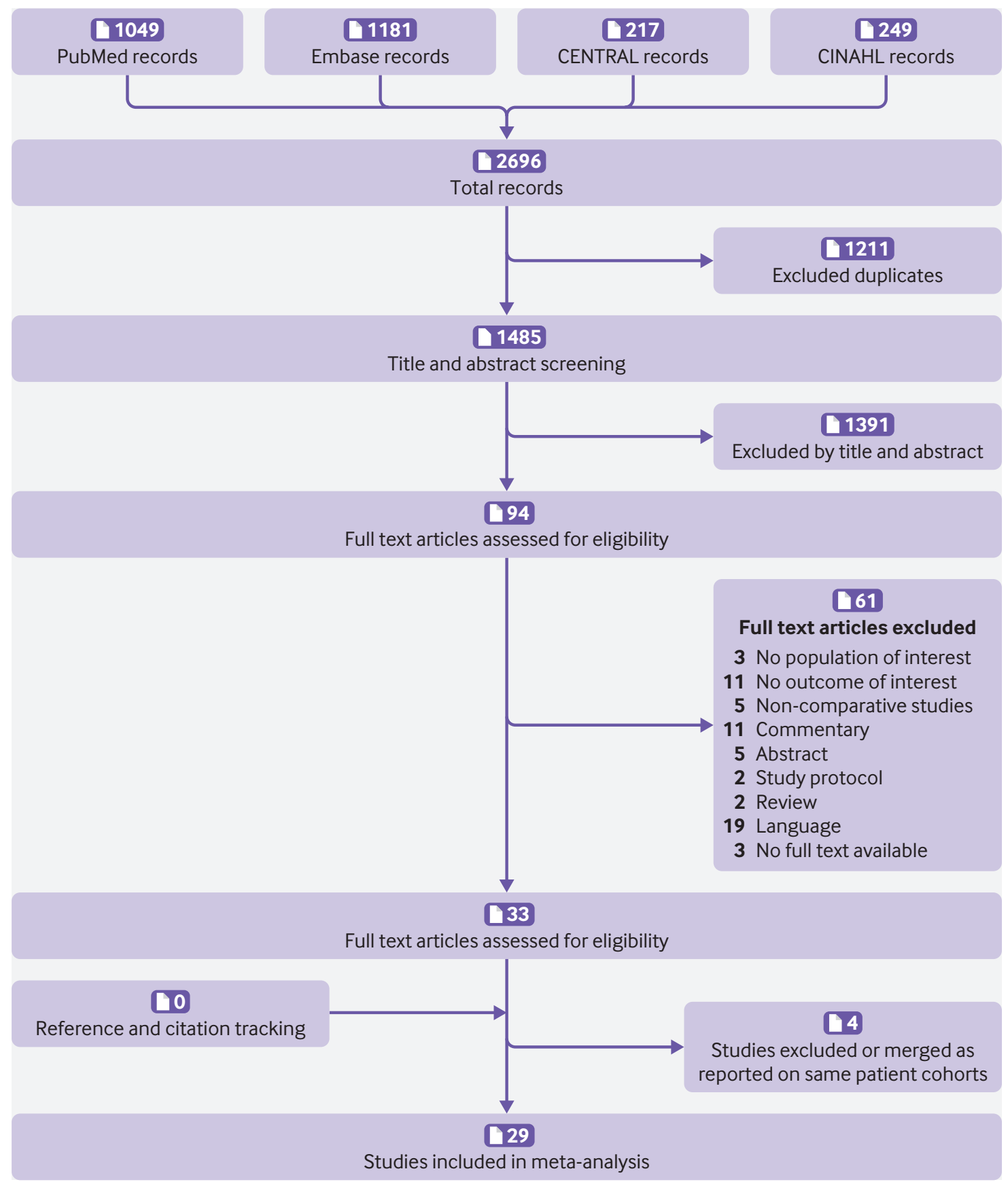

Fig 1 | PRISMA flow diagram representing search and selection of studies comparing operative versus nonoperative treatment of Achilles tendon ruptures

nonoperatively. The weighted mean age was 40 years in both treatment groups, and 779 (83\%) males were included. The operative method was open surgery in nine studies and minimally invasive surgery in one study.

The 19 observational studies-three prospective and 16 retrospective cohort studies-included 14918 (94\%) patients. Operative treatment was performed in 8906 patients, and 6012 were treated nonoperatively. The weighted mean age in the studies was 42 (range 17-86) years, 40 years in the operative group and 44 years in the nonoperative group, and 11000 (74\%) patients were male. The operative method was open surgery in nine studies, unclear in four studies, and a combination of open and minimally invasive surgery in six studies.

\section{Quality assessment}

The overall mean MINORS score was 14.3 (SD 5.2; range 5-23). The mean MINORS score for the RCTs was 20.3 (2.6; 16-23). The mean MINORS score for the observational studies was 11.2 (2.8; 5-16), 14 (2; 12 16) for the prospective cohort studies and 10.6 (2.6; 5-15) for the retrospective cohort studies. The details and distribution of MINORS scores are provided in supplementary table D. 


\begin{tabular}{|c|c|c|c|c|c|c|c|c|c|c|c|}
\hline \multirow[b]{2}{*}{ Study and year } & \multirow{2}{*}{$\begin{array}{l}\text { Study } \\
\text { period }\end{array}$} & \multirow[b]{2}{*}{ Country } & \multirow{2}{*}{$\begin{array}{l}\text { Overall } \\
\text { No }\end{array}$} & \multicolumn{2}{|c|}{ Number } & \multicolumn{2}{|c|}{ Mean (SD or range) age, years } & \multicolumn{2}{|c|}{ Sex (female/male) } & \multicolumn{2}{|c|}{$\begin{array}{l}\text { Mean (SD or range) } \\
\text { follow-up, months }\end{array}$} \\
\hline & & & & OP & NON & OP & NON & OP & NON & OP & NON \\
\hline \multicolumn{12}{|c|}{ Randomised controlled trials } \\
\hline Cetti et al, 1993 & $1982-84$ & Denmark & 111 & 56 & 55 & $37.2(21-62)$ & $37.8(21-65)$ & $9 / 47$ & $10 / 45$ & 12 & 12 \\
\hline Keating et al, 2011 & 2000-04 & UK & 80 & 39 & 41 & $41.2(27-59)$ & $39.5(21-58)$ & $11 / 28$ & $9 / 32$ & 12 & 12 \\
\hline Lantto et al, 2016 & 2009-13 & Finland & 60 & 32 & 28 & $40(27-57)$ & $39(28-60)$ & $2 / 30$ & $3 / 25$ & 18 & 18 \\
\hline Metz et al, 2008 & 2004-05 & Netherlands & 83 & 42 & 41 & $40(23-63)$ & $41(25-62)$ & $11 / 31$ & $6 / 35$ & 12 & 12 \\
\hline Möller et al, 2001 & 1995-97 & Sweden & 112 & 59 & 53 & $39.6(21-63)$ & $38.5(26-59)$ & $8 / 51$ & $5 / 48$ & 24 & 24 \\
\hline $\begin{array}{l}\text { Nilsson-Helander et } \\
\text { al, } 2010\end{array}$ & 2004-07 & Sweden & 97 & 49 & 48 & $40.9(8.8)$ & $41.2(9.5)$ & $9 / 40$ & $9 / 39$ & 12 & 12 \\
\hline Nistor et al, 1981 & 1973-77 & Sweden & 107 & 46 & 61 & \multicolumn{2}{|c|}{ Overall 41 (21-77) } & \multicolumn{2}{|l|}{ Overall 11/96 } & \multicolumn{2}{|c|}{ Overall $30(12-60)$} \\
\hline Olsson et al, 2013 & 2009-10 & Sweden & 100 & 49 & 51 & $39.8(8.9)$ & $39.5(9.7)$ & $10 / 39$ & $4 / 47$ & 12 & 12 \\
\hline Twaddle et al, 2007 & 1997-2002 & New Zealand & 50 & 25 & 25 & 41.8 & 40.3 & $6 / 14^{*}$ & $8 / 14^{*}$ & 12 & 12 \\
\hline Willits et al, 2010 & 2000-05 & Canada & 144 & 72 & 72 & 39.7 (11) & $41.1(8.0)$ & $13 / 59$ & $13 / 59$ & 24 & 24 \\
\hline \multicolumn{12}{|l|}{ Observational studies } \\
\hline Bergkvist et al, 2012 & $2002-06$ & Sweden & 487 & 220 & 267 & $43(11)$ & $47(14)$ & \multicolumn{2}{|l|}{ Overall 78/409 } & \multicolumn{2}{|c|}{ Overall 43 (12-97) } \\
\hline Carden et al, 1987 & 1969-81 & UK & 71 & 35 & 36 & $42.7(26-68)$ & $43(22-70)$ & $10 / 26$ & $12 / 25$ & $\begin{array}{l}48 \\
(12-204) \\
\end{array}$ & $\begin{array}{l}64 \\
(12-120) \\
\end{array}$ \\
\hline Costa et al, $2006+$ & 2001-02 & UK & 96 & 48 & 48 & $42(28-69)$ & $53(21-79)$ & $7 / 40^{*}$ & $16 / 32$ & 12 & 12 \\
\hline Cukelj et al, 2015 & $1998-2013$ & Croatia & 90 & 60 & 30 & $34.8(4.7$ & $35.1(4.7)$ & $9 / 51$ & $9 / 21$ & \multicolumn{2}{|l|}{ Overall 12} \\
\hline Ebinesan et al, 2008 & 2001-03 & UK & 63 & 51 & 12 & 44.8 & 52.1 & $14 / 37$ & $6 / 6$ & \multicolumn{2}{|l|}{ NA } \\
\hline Fahlström et al, 1998 & 1990-94 & Sweden & 31 & 22 & 9 & $34.6(23-50)$ & $39.4(28-51)$ & \multicolumn{2}{|l|}{ Overall 4/27 } & \multicolumn{2}{|c|}{ Overall 39 (16-67) } \\
\hline Grubor et al, 2012 & $2003-10$ & Bosnia & 42 & 34 & 8 & NA & & \multicolumn{2}{|l|}{ Overall 5/37 } & \multicolumn{2}{|c|}{ Overall 12} \\
\hline $\begin{array}{l}\text { Gwynne-Jones } \\
\text { et al, } 2011\end{array}$ & 1999-2008 & New Zealand & 363 & 143 & 220 & 37.4 & 40.9 & $59 / 84$ & $107 / 113$ & NA & \\
\hline Jaakkola et al, 2001 & 1985-99 & USA & 73 & 35 & 38 & $37.3(25-64)$ & $38.0(21-62)$ & $3 / 32$ & $6 / 32$ & 43 & 54 \\
\hline Jackson et al, 2013† & $2002-08$ & UK & 80 & 29 & 51 & $37(24-55)$ & $47(27-80)$ & $3 / 26$ & $16 / 35$ & NA & \\
\hline Kotnis et al, $2006 \dagger$ & 2000-05 & UK & 125 & 67 & 58 & $41.0(26-80)$ & $43.9(26-85)$ & $19 / 48$ & $18 / 40$ & 12 & 12 \\
\hline Lim et al, 2017 & NA & New Zealand & 200 & 99 & 101 & 40.1 & 42 & $21 / 41^{*}$ & $32 / 38^{\star}$ & \multicolumn{2}{|c|}{ Overall 78 (24-156) } \\
\hline Miller et al, 2005 & 1990-96 & UK & 172 & 140 & 32 & 45 & 49 & $23 / 117$ & $11 / 21$ & \multicolumn{2}{|c|}{ Overall 95 (53) } \\
\hline Nestorson et al, 2000 & 1992-97 & Sweden & 24 & 14 & 10 & $72(65-79)$ & $71(65-86)$ & $3 / 11$ & $1 / 9$ & \multicolumn{2}{|c|}{ Overall 39 (13-65) } \\
\hline Rajasekar et al, 2005 & $1997-2001$ & UK & 35 & 21 & 14 & NA & & Overall 10/25 & & \multicolumn{2}{|c|}{ Overall 24 (9-48) } \\
\hline Renninger et al, 2016 & 2011-14 & USA & 57 & 27 & 30 & $32.3(25-40)$ & $29.7(23-44)$ & $0 / 27$ & $0 / 30$ & \multicolumn{2}{|l|}{ Overall 10} \\
\hline $\begin{array}{l}\text { Van der Linden } \\
\text { et al, } 2004\end{array}$ & $1990-2001$ & Netherlands & 292 & 212 & 80 & $37(9.4)$ & $42(12)$ & $58 / 154$ & $21 / 59$ & \multicolumn{2}{|c|}{ Overall 72 (36) } \\
\hline Wang et al, 2015 & 2007-11 & USA & 12570 & 7625 & 4945 & NA & & $1514 / 6111$ & $1737 / 3208$ & NA & \\
\hline Weber et al, 2003 & 1993-98 & Switzerland & 47 & 24 & 23 & $38(28-51)$ & $39(17-55)$ & $4 / 13^{*}$ & $8 / 15$ & $\begin{array}{l}49 \\
(30-79)\end{array}$ & $\begin{array}{l}23 \\
(12-42)\end{array}$ \\
\hline
\end{tabular}

\section{Primary outcome measure}

Re-rupture rate

Re-rupture rate was reported in all 29 studies. The overall pooled effect showed that operative treatment was associated with a significant reduction in rerupture rate compared with nonoperative treatment (risk ratio $0.43,95 \%$ confidence interval 0.31 to 0.60 ; $\mathrm{P}<0.001 ; \mathrm{I}^{2}=22 \%$ ) (fig 2 ). The pooled effect of RCTs showed a risk ratio of $0.40(0.24$ to 0.69 ; $\mathrm{P}<0.001$; $\left.\mathrm{I}^{2}=0 \%\right)$. The pooled effect of observational studies showed a risk ratio of $0.42(0.28$ to $0.64 ; \mathrm{P}<0.001$; $\mathrm{I}^{2}=31 \%$ ). Re-rupture occurred in $2.3 \%$ of patients after operative treatment compared with $3.9 \%$ after nonoperative treatment (risk difference 1.6\%). We found no significant difference in effect estimates from RCTs and observational studies (test for subgroup differences: $\mathrm{P}=0.91 ; \mathrm{I}^{2}=0 \%$ ). There was no visual asymmetry in the funnel plot (supplementary figure A).
The Begg rank correlation test $(\mathrm{P}=0.66)$ and Egger linear regression test $(\mathrm{P}=0.16)$ indicated no evidence of publication bias.

\section{Secondary outcome measures \\ Complication rate}

Complication rate was reported in 26 (90\%) studies-10 RCTs and 16 observational studies. The overall pooled effect showed a risk ratio of 2.76 (1.84 to 4.13; $\mathrm{P}<0.001 ; \mathrm{I}^{2}=45 \%$ ) in favour of nonoperative treatment compared with operative treatment (fig 3). The pooled effect of RCTs showed a risk ratio of 3.26 (1.26 to 8.41; $\mathrm{P}=0.01 ; \mathrm{I}^{2}=74 \%$ ). The pooled effect of observational studies showed a risk ratio of 2.93 (2.28 to 3.75; $\left.\mathrm{P}<0.001 ; \mathrm{I}^{2}=0 \%\right)$. The incidence of complications was 4.9\% after operative treatment compared with $1.6 \%$ after nonoperative treatment (risk difference 3.3\%). Table 2 shows the classification and incidence of 


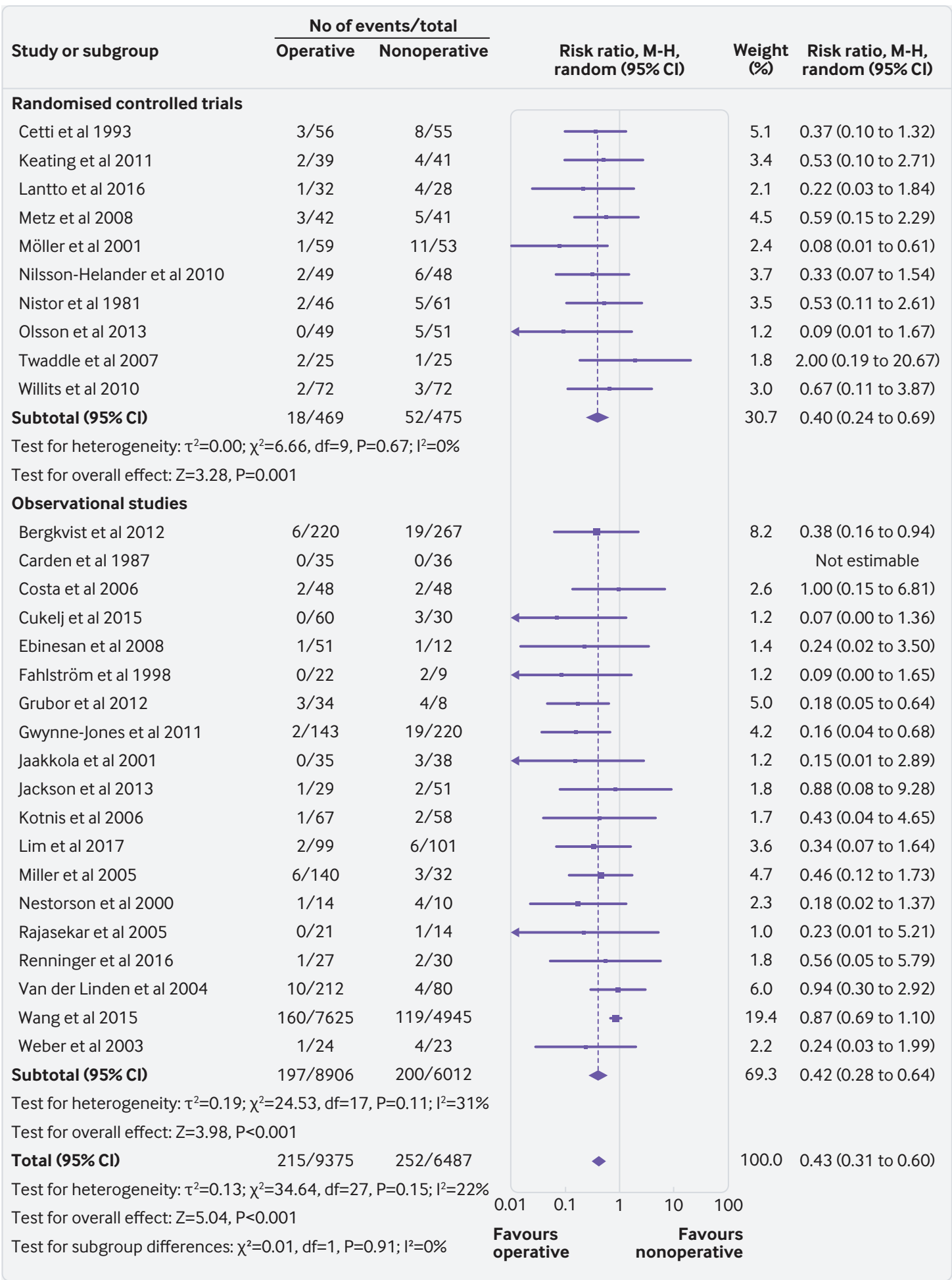

Fig 2 | Forest plot of re-rupture rate in meta-analysis of Achilles tendon ruptures. $\mathrm{M}-\mathrm{H}=$ Mantel-Haenszel

complications. The main complication after operative treatment was infection, which occurred in $2.8 \%$ of patients. The main complication after nonoperative treatment was deep vein thrombosis, which occurred in $1.2 \%$ of patients compared with $1.0 \%$ after operative treatment. We found no significant difference between effect estimates from RCTs and observational studies (test for subgroup differences: $\mathrm{P}=0.83 ; \mathrm{I}^{2}=0 \%$ ).
There was no visual asymmetry in the funnel plot (supplementary figure B). The Begg rank correlation test $(\mathrm{P}=0.50)$ and Egger linear regression test $(\mathrm{P}=0.11)$ indicated no evidence of publication bias.

\section{Functional outcome}

Short term functional outcome assessed according to the ATRS score was reported in three (10\%) studies. 
Nilsson-Helander et al reported a median ATRS score of 75 (range 31-100) in the operative group and $90(31-100)$ in the nonoperative group. ${ }^{58}$ Olsson et al reported a mean ATRS score of 82 (SD 20) in the operative group compared with 80 (23) in the nonoperative group. ${ }^{59}$ In both RCTs, the differences found were non-significant. The observational study by Jackson et al reported a statistical significant difference in median ATRS score-94 (range 23$100)$ in the operative group and 84 (25-100) in the nonoperative group. ${ }^{34}$
Long term functional outcome using the ATRS score was assessed in two observational studies. Bergkvist et al reported a mean ATRS score of 83 (SD 19) in the operative group and 78 (22) in the nonoperative group. ${ }^{36}$ Lim et al reported a mean ATRS score of 85 in both groups. ${ }^{44}$ No significant difference was found in either study. We did not pool functional outcome data owing to a wide variety in ATSR score reports and insufficient information to convert data. Descriptive details on functional outcome measures are provided in supplementary table E.

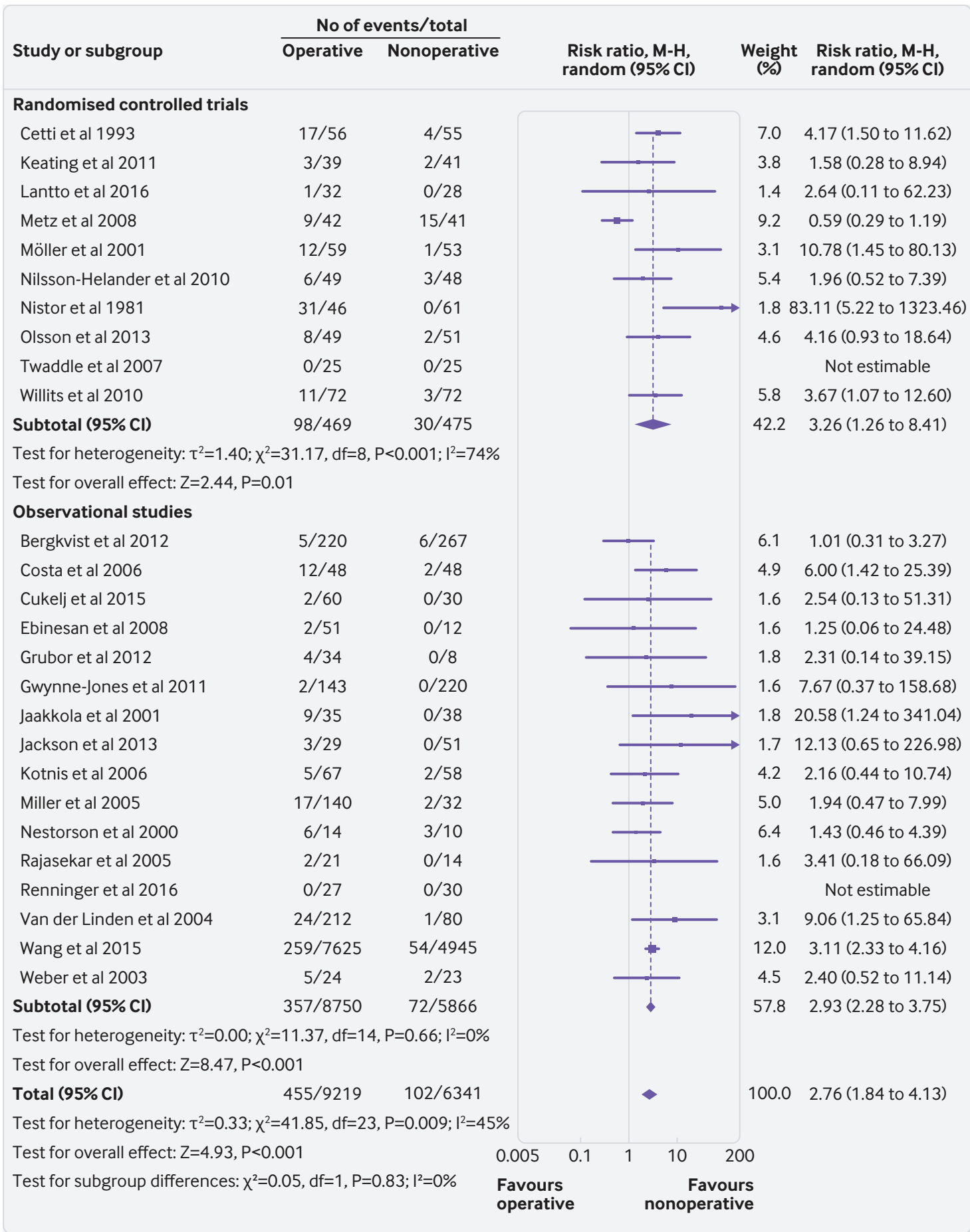

Fig 3 | Forest plot of complication rate in meta-analysis of Achilles tendon ruptures. $\mathrm{M}-\mathrm{H}=$ Mantel-Haenszel 


\begin{tabular}{lllll}
\hline \multirow{2}{*}{$\begin{array}{l}\text { Table } 2 \text { | Number and incidence of complications in studies included in meta-analysis of Achilles tendon ruptures } \\
\text { Complication classification }\end{array}$} & Operative treatment & Nonoperative treatment \\
\cline { 2 - 5 } & No & Incidence (\%) & No & Incidence (\%) \\
\hline Pulmonary embolism & 2 & 0.02 & 2 & 0.03 \\
\hline Deep vein thrombosis & 89 & 0.97 & 74 & 1.17 \\
\hline Wound/skin infection & 258 & 2.80 & 1 & 0.02 \\
\hline Sural nerve injury & 39 & 0.42 & 5 & 0.08 \\
\hline Chronic pain & 3 & 0.03 & 2 & 0.03 \\
\hline Scar/skin adhesion & 35 & 0.38 & 15 & 0.24 \\
\hline Wound dehiscence & 8 & 0.09 & 0 & 0 \\
\hline Not specified/other & 21 & 0.23 & 3 & 0.05 \\
\hline Total & 455 & 4.94 & 102 & 1.61 \\
\hline
\end{tabular}

Return to sports and work

Return to sports was reported by four (14\%) studies-one RCT and three observational studies (supplementary table E). The mean time varied between six and nine months after operative treatment and between six and eight months after nonoperative treatment. We could not pool data on return to sports in a meta-analysis, as only one study reported a mean and standard deviation.

Return to work was reported in nine (31\%) studiesfourRCTsandfiveobservationalstudies(supplementary table E). The outcome data of six studies could not be pooled owing to insufficient reporting of information. The pooled effect estimates of three studies-two RCTs and one observational study-showed no significant mean difference between operative and nonoperative treatment groups (supplementary figure C).

\section{Primary sensitivity analysis}

Weight bearing status

Early (four weeks or less) weight bearing status was reported in nine (31\%) studies-five RCTs and four observational studies. The overall pooled effect showed a significant reduction in re-rupture rate after operative treatment compared with nonoperative treatment in the early (four weeks or less) full weight bearing studies (risk ratio $0.49,0.26$ to $0.93 ; \mathrm{P}=0.03$; $\mathrm{I}^{2}=9 \%$ ) (supplementary figure D). Late (more than four weeks) weight bearing status was reported in 15 (52\%) studies-four RCTs and 11 observational studies. The overall pooled effect of the late (more than four weeks) full weight bearing studies also showed a significant reduction in re-rupture rate in favour of operative treatment (risk ratio $0.33,0.21$ to $0.50 ; \mathrm{P}<0.001$; $\mathrm{I}^{2}=0 \%$ ) (supplementary figure $\mathrm{E}$ ).

\section{Accelerated functional rehabilitation}

Accelerated functional rehabilitation with early range of motion was performed in six (21\%) studies-three RCTs and three observational studies. The overall pooled effect showed no significant difference between operative and nonoperative treatment regarding rerupture rate (risk ratio $0.60,0.26$ to $1.37 ; \mathrm{P}=0.23$; $\mathrm{I}^{2}=0 \%$ ) (fig 4).

\section{Secondary sensitivity analyses}

Table 3 shows the results of the secondary sensitivity analyses. Re-rupture rate was reported in 17 (59\%) high quality studies-10 RCTs and seven observational studies. The overall pooled effect showed that operative treatment was associated with a significant reduction in re-rupture rate compared with nonoperative treatment (risk difference 5.1\%; risk ratio 0.44, 0.30 to $0.64 ; \mathrm{P}<0.001 ; \mathrm{I}^{2}=0 \%$ ) (supplementary figure $\mathrm{F}$ ). Re-rupture rate was reported in 14 studies (48\%) with a study period after the year 2000-six RCTs and eight observational studies. The overall pooled effect showed a significant reduction in re-rupture rate after operative treatment compared with nonoperative treatment (risk difference $0.9 \%$; risk ratio $0.59,0.42$ to $0.83 ; \mathrm{P}=0.002 ; \mathrm{I}^{2}=10 \%$ ) (supplementary figure $\mathrm{G}$ ).

Complication rate was reported in 16 (55\%) high quality studies-10 RCTs and six observational studies. The overall pooled effect showed a risk ratio of 2.72 ( 1.44 to $5.12 ; \mathrm{P}=0.002 ; \mathrm{I}^{2}=62 \%$ ) in favour of nonoperative treatment compared with operative treatment (risk difference 8.8\%) (supplementary figure $\mathrm{H})$. Complication rate was reported in 14 (48\%) studies with a study period after the year 2000-six RCTs and eight observational studies. The overall pooled effect showed a risk ratio of 2.15 (1.28 to 3.60; $\mathrm{P}=0.004 ; \mathrm{I}^{2}=52 \%$ ) in favour of nonoperative treatment compared with operative treatment (risk difference 2.4\%) (supplementary figure I).

\section{Discussion}

This systematic review and meta-analysis, including both RCTs and observational studies, compared outcomes after operative versus nonoperative treatment of acute Achilles tendon ruptures. The pooled effect estimate showed that operative treatment was associated with a significant reduction in rerupture rate compared with nonoperative treatment. However, operative treatment resulted in a significantly higher rate of other complications. Sensitivity analyses showed a similar reduction in re-rupture rate after both early and late full weight bearing in favour of operative treatment compared with nonoperative treatment. However, we found no significant difference in rerupture rate if accelerated functional rehabilitation with early range of motion was used. Sensitivity analyses with high quality studies and studies with a study period after the year 2000 also showed operative treatment to be associated with a significant reduction in re-rupture rate but a higher risk of other complications. We found no significant difference in 


\begin{tabular}{|c|c|c|c|c|c|}
\hline \multirow[b]{2}{*}{ Study or subgroup } & \multicolumn{2}{|c|}{ No of events/total } & \multirow[b]{2}{*}{$\begin{array}{l}\text { Risk ratio, } \mathrm{M}-\mathrm{H}, \\
\text { random }(95 \% \mathrm{Cl})\end{array}$} & \multirow[b]{2}{*}{$\begin{array}{l}\text { Weight } \\
(\%)\end{array}$} & \multirow[b]{2}{*}{$\begin{array}{l}\text { Risk ratio, } \mathrm{M}-\mathrm{H}, \\
\text { random }(95 \% \mathrm{Cl})\end{array}$} \\
\hline & Operative & Nonoperative & & & \\
\hline \multicolumn{6}{|l|}{ Randomised controlled trials } \\
\hline Nilsson-Helander et al 2010 & $2 / 49$ & $6 / 48$ & $\longrightarrow$ & 28.5 & 0.33 (0.07 to 1.54$)$ \\
\hline Twaddle et al 2007 & $2 / 25$ & $1 / 25$ & & 12.5 & 2.00 (0.19 to 20.67$)$ \\
\hline Willits et al 2010 & $2 / 72$ & $3 / 72$ & & 22.1 & $0.67(0.11$ to 3.87$)$ \\
\hline Subtotal $(95 \% \mathrm{Cl})$ & $6 / 146$ & $10 / 145$ & & 63.1 & 0.60 (0.21 to 1.70$)$ \\
\hline \multicolumn{6}{|c|}{ Test for heterogeneity: $\tau^{2}=0.00 ; \chi^{2}=1.63, d f=2, P=0.44 ; I^{2}=0 \%$} \\
\hline \multicolumn{6}{|c|}{ Test for overall effect: $Z=0.96, P=0.34$} \\
\hline \multicolumn{6}{|l|}{ Observational studies } \\
\hline Jackson et al 2013 & $1 / 29$ & $2 / 51$ & & 12.3 & 0.88 (0.08 to 9.28$)$ \\
\hline Kotnis et al 2006 & $1 / 67$ & $2 / 58$ & & 12.1 & 0.43 (0.04 to 4.65$)$ \\
\hline Renninger et al 2016 & $1 / 27$ & $2 / 30$ & & 12.5 & 0.56 (0.05 to 5.79$)$ \\
\hline Subtotal $(95 \% \mathrm{Cl})$ & $3 / 123$ & $6 / 139$ & & 36.9 & 0.60 (0.15 to 2.33$)$ \\
\hline \multicolumn{6}{|c|}{ Test for heterogeneity: $\tau^{2}=0.00 ; \chi^{2}=0.18, d f=2, P=0.91 ; I^{2}=0 \%$} \\
\hline \multicolumn{6}{|c|}{ Test for overall effect: $Z=0.74, P 0.46$} \\
\hline Total $(95 \% \mathrm{Cl})$ & $9 / 269$ & $16 / 284$ & $\sim$ & 100.0 & $0.60(0.26$ to 1.37$)$ \\
\hline \multicolumn{6}{|c|}{ Test for heterogeneity: $\tau^{2}=0.00 ; \chi^{2}=1.80, d f=5, P=0.88 ; I^{2}=0 \%$} \\
\hline Test for overall effect: $Z=1.21$, & & 0.01 & 0.1 & 100 & \\
\hline Test for subgroup differences: & $00, d f=1, P=C$ & $99 ; 1^{2}=0 \%$ & nonor & $\begin{array}{l}\text { ours } \\
\text { tive }\end{array}$ & \\
\hline
\end{tabular}

Fig 4 | Forest plot of re-rupture rate in studies that included accelerated functional rehabilitation in meta-analysis of Achilles tendon ruptures. $\mathrm{M}-\mathrm{H}=$ Mantel-Haenszel

effect estimates from RCTs and observational studies, for either re-rupture rate or complication rate.

\section{Comparison with previous findings}

Operative treatment reduces the risk of re-rupture compared with nonoperative treatment, but it also results in a higher risk of other complications. These findings are in accordance with those of previous metaanalyses. $^{346}$ Our review included 10 RCTs with a total of 944 patients, which resulted in an increased number of patients available for analyses, thus exceeding previous meta-analyses. Furthermore, the inclusion of observational studies resulted in an additional 14918 patients for analyses. The previous metaanalyses reported a risk difference in re-rupture rate varying from $5 \%$ to $7 \%$ and a risk difference of other complications varying from $16 \%$ to $21 \% .^{3-6}$ However, with the addition of observational studies, this review shows that differences between treatment groups are small, with a risk difference in re-rupture rate of 1.6\% and a risk difference of $3.3 \%$ for other complications.

Functional outcome measures included the ATRS score, return to sports, and return to work. The ATRS score is the most commonly used patient reported instrument to evaluate limitations after treatment for an acute Achilles tendon rupture. ${ }^{2}$ ATRS scores were not pooled in this study, but most studies showed no significant difference in ATRS score between the operative and nonoperative treatment groups. Resumption of sports was reported by only four studies; the results indicate no difference between operative treatment (six to nine months) and nonoperative treatment (six to eight months). The pooled effect of return to work showed no significant difference between treatment groups. Wilkins et al pooled return to work data from four studies and also found no statistical significant difference. ${ }^{5}$ Soroceanu et al reported a statistically significant difference with the pooled data from four studies; operatively treated patients returned to work 19 days earlier than nonoperatively treated patients $(\mathrm{P}=0.0014){ }^{6}$ Wilkins et al included return to work data in their pooled results from the studies by Nistor et al and Cetti et al. ${ }^{5257}$ In our study, we did not use the return to work data from these two studies owing to reporting of mean and range and the absence of standard deviations. Soroceanu et al also included the study by Cetti et al, as well as the study by Majewski et al, ${ }^{62} 62$ which we excluded

\begin{tabular}{|c|c|c|c|c|c|c|c|c|c|c|}
\hline \multirow[b]{2}{*}{ Studies } & \multicolumn{5}{|c|}{ Re-rupture } & \multicolumn{5}{|c|}{ Complication } \\
\hline & No & RD (\%) & $\mathrm{RR}(95 \% \mathrm{Cl})$ & P value & $12(\%)$ & No & RD (\%) & $\mathrm{RR}(95 \% \mathrm{Cl})$ & $P$ value & $12(\%)$ \\
\hline High quality studies & 17 & 5.1 & $0.44(0.30$ to 0.64$)$ & $<0.001$ & 0 & 16 & 8.8 & 2.72 (1.44 to 5.12$)$ & 0.002 & 62 \\
\hline $\begin{array}{l}\text { Study period ( } 2000 \\
\text { or after) }\end{array}$ & 14 & 0.9 & 0.59 (0.42 to 0.83) & 0.002 & 10 & 14 & 2.4 & 2.15 (1.28 to 3.60$)$ & 0.004 & 52 \\
\hline
\end{tabular}


as it was in a language other than English. However, both our meta-analysis and the studies by Wilkins et al and Soroceanu et al are limited by the number of included patients in the return to work subgroup analyses. ${ }^{5}$ Unfortunately, accurate comparison of functional outcome measures remains difficult owing to differences in protocols, patient oriented outcome measures, duration of follow-up, and presentation of data.

We found a lower re-rupture rate after both early and late full weight bearing in favour of operative treatment; this is in contrast to a previous meta-analysis by Van der Eng et al, ${ }^{63}$ which found no difference in re-rupture rate. The previous meta-analysis could be limited by the number of included patients in the subgroup analyses. In our review, with the addition of observational studies, sensitivity analysis showed a significant difference in re-rupture rate after both early and late full weight bearing in favour of operative treatment. However, regardless of re-rupture rate, timing of weight bearing might influence other outcome measures as shown in different lower extremity injuries. De Boer et al found that early weight bearing regimens did not negatively affect functional outcome after treatment for displaced intra-articular calcaneal fractures. ${ }^{64}$ Previously, Smeeing et al showed that early weight bearing tended to accelerate return to work and daily activities compared with late weight bearing, after internal fixation of ankle fractures. ${ }^{65}$ Eliasson et al evaluated tendon elongation, mechanical properties, and functional outcomes during the first 12 months after operative treatment of acute Achilles tendon ruptures. ${ }^{66}$ However, they found that different rehabilitation regimens did not affect the outcome measures. Further research could focus on the effect of early weight bearing and long term functional outcome after treatment of Achilles tendon ruptures.

Soroceanu et al found no significant difference in re-rupture rate in their subgroup analysis if functional rehabilitation with early range of motion was used (risk difference $1.7 \%$; $\mathrm{P}=0.45) .{ }^{6}$ However, they did not define the specific inclusion criteria and definition of early range of motion and functional rehabilitation. Unfortunately, evaluation of the effect of accelerated functional rehabilitation remains difficult owing to use of a wide variety of definitions and protocols. Our review found no significant difference in re-rupture rate if accelerated functional rehabilitation with early range of motion within three weeks was used after nonoperative treatment. These findings indicate that nonoperative management is acceptable for acute Achilles tendon ruptures, if patients are instructed and monitored according to a standardised rehabilitation protocol. However, both our review and the study by Soroceanu et al could be limited by the number of included patients in the subgroup analyses. ${ }^{6}$

The sensitivity analyses including high quality studies resulted in similar risk ratios and significance levels for re-rupture and complication rate. The results showed a risk difference of $5.1 \%$ for re-rupture rate, comparable to previous results of meta-analyses of
RCTs alone. However, the risk difference of other complications $(8.8 \%)$ in the high quality sensitivity analysis was still considerably lower than in previous reports. This difference in other complications could be attributable to the inclusion of studies with both open and minimally invasive surgical techniques. The complication sensitivity analysis with high quality studies included one RCT with minimally invasive surgery and three observational studies that included both open and minimally invasive surgery. A metaanalysis by Yang et al, ${ }^{67}$ including five RCTs and four cohort studies, found a significantly lower rate of deep infection with percutaneous treatment $(0.6 \%)$ than with open treatment $(3.6 \%)(\mathrm{P}=0.04)$. However, the authors reported no significant difference in the rate of rerupture between percutaneous and open treatment. ${ }^{67}$

The sensitivity analyses including studies with a study period after the year 2000 showed similar risk ratios and significance levels regarding re-rupture and complication rate. However, the risk differences between treatment groups were smaller than in all other analyses. The study period sensitivity analyses included one RCT with minimally invasive surgery and four observational studies that included both open and minimally invasive surgery. These findings might indicate an overall reduction in complications after treatment of Achilles tendon ruptures due to the development of new rehabilitation protocols and operative techniques, regardless the use of operative or nonoperative treatment. However, it should be noted that both the level of high quality studies and the study period were arbitrarily chosen.

We found no difference in pooled effect estimates from RCTs and observational studies. This is in line with previous reports showing that differences in effect estimates between RCTs and observational studies are small. $^{8911131517}$ Observational studies, however, have also been associated with an overestimation of treatment effects compared with RCTs. ${ }^{68}{ }^{69}$ Hemkens et al assessed the difference in treatment effect estimates for mortality between observational studies and RCTs. ${ }^{69}$ They evaluated 16 observational studies and 36 subsequent RCTs investigating the same clinical questions. Overall, observational studies significantly overestimated the effects of treatment compared with RCTs. ${ }^{69}$ This overestimation of treatment effects could be explained by the effects of bias and confounding in observational studies. ${ }^{70}$ However, overestimates by observational studies could also be explained by the potential selection bias in RCTs. RCTs require strict conditions such as selection of participants, inclusion/exclusion criteria, randomisation method, and outcome measurements. The patient population in daily clinical practice can differ from the often highly selected patient populations in RCTs, which could be the reason for the discrepancy between treatment effects. ${ }^{71}{ }^{72}$ Nevertheless, observational studies increase sample size, which could lead to the evaluation of small treatment effects and infrequent outcome measures. Furthermore, the addition of observational studies might provide insight into a 
variety of populations and long term effects. These results could improve the representation of daily clinical practice, with various levels of surgical experience and differences in operative techniques, provided that confounding has been adequately addressed. ${ }^{12}{ }^{13}$ In this meta-analysis, pooled effect estimates obtained from RCTs and observational studies were similar. Several orthopaedic trauma meta-analyses including both RCTs and observational studies have shown high quality observational studies to result in similar treatment effects to RCTs. ${ }^{15-17}$ These findings indicate that the effect of potentially unmeasured confounding in high quality observational studies seems relatively small, emphasising the possible benefits of combining different study designs for the evaluation of objective outcome measures after surgical treatment.

\section{Limitations of this study}

Several potential limitations in this review need to be considered. Firstly, results might be influenced by missing articles. However, in addition to the extensive electronic database search, funnel plots did not indicate evidence for publication bias. Three studies could not be obtained in full text, but these articles were all published before $1996{ }^{26-28}$ Secondly, the methodological quality of included studies was assessed by the MINORS criteria, which do not differentiate between randomised and non-randomised studies. However, the MINORS criteria were externally validated using RCTs and are able to distinguish adequately between study designs, as well designed randomised trials score higher than well designed non-randomised studies. ${ }^{21}$ The incidence of complications could be affected by the use of different treatment protocols. Five studies mentioned the use of prophylaxis for deep vein thrombosis. ${ }^{51} 53565859$ However, descriptions were not comprehensive and the duration and types of prophylaxis varied widely. Finally, sensitivity analyses for the evaluation of weight bearing status and accelerated rehabilitation were performed using data from both RCTs and observational studies. However, the primary analysis showed no significant difference in effect estimates between the two study designs in terms of re-rupture rate.

\section{Implications for future research}

Operative treatment of acute Achilles tendon ruptures reduces the risk of re-rupture compared with nonoperative treatment, although the incidence of re-ruptures is low and differences are small $(2.3 \%$ $v$ 3.9\%). Operative treatment results in a higher risk of other complications compared with nonoperative treatment, mostly attributable to the increased risk of infection. Nonoperative treatment might be the preferred treatment for acute Achilles tendon rupture, owing to the higher risk of other complications after operative treatment and the relative small benefit in rerupture rate. However, patient specific factors should always be taken into consideration and patients should be counselled about the incidence of complications.

Unfortunately, comparison of the literature remains difficult owing to a wide variety of rehabilitation protocols, weight bearing restrictions, treatment modalities, patient oriented outcome measures, and duration of follow-up. The discordance among studies makes comparisons between treatment modalities difficult, indicating a substantial need for further research. We suggest future research to focus on the effect of comorbidities on the success of treatment for Achilles tendon rupture. Studies could compare outcomes according to different age groups and evaluate effects in a variety of populations such as in patients with immunosuppression, diabetes mellitus, increased body mass index, neuropathy, peripheral vascular disease, and dermatological disorders. Furthermore, future studies should strive to determine the optimal treatment for acute Achilles tendon ruptures on the basis of patients' expectations. Operative treatment is associated with complications inherent to the treatment itself, such as infection. However, athletic people may prefer operative treatment to enhance and expedite their outcomes, whereas a sedentary person with limited functional outcome expectations may prefer nonoperative treatment. ${ }^{3}$ We believe that more data are needed for the development of a shared decision making algorithm to guide surgeons and physicians regarding the most appropriate treatment option for each individual patient.

\section{Conclusions}

In this meta-analysis, operative treatment of acute Achilles tendon ruptures reduced the risk of re-rupture compared with nonoperative treatment. However, rerupture rates are low and differences between treatment groups are small, with a risk difference of $1.6 \%$. Operative treatment results in a higher risk of other complications, with a risk difference of 3.3\%, mostly due to the increased risk of infection. Patients should be counselled about complications, and the final decision for operative or nonoperative management should be based on patient specific factors and shared decision making. Further research is needed for the development of a shared decision making algorithm. Moreover, this review emphasises the potential benefits of adding high quality observational studies in metaanalyses to complement RCTs for the evaluation of objective outcome measures after surgical treatments.

\section{AUTHOR AFFILIATIONS}

${ }^{1}$ Department of Surgery, University Medical Center Utrecht, 3508 GA Utrecht, Netherlands

${ }^{2}$ Department of Orthopedic Surgery, Harvard Medical School Orthopedic Trauma Initiative, Massachusetts General Hospital, Boston, MA, USA

${ }^{3}$ Department of Surgery, Diakonessenhuis Hospital, Utrecht, Netherlands

${ }^{4}$ Department of Surgery, Academic Medical Center, Amsterdam, Netherlands

${ }^{5}$ Department of Surgery, St. Antonius Hospital, Nieuwegein, Netherlands

${ }^{6}$ Department of Orthopedic Surgery, Spaarne Hospital, Hoofddorp, Netherlands

${ }^{7}$ Department of Clinical Epidemiology, Leiden University Medical Center, Leiden, Netherlands 
Contributors: RMH and RHHG conceived and designed the study. YO RHHG, RBB, and RMH acquired, analysed, and interpreted the data. YO drafted the first version of the manuscript. YO, OvdM, RHHG, RBB, $\mathrm{MH}$, and RMH drafted the subsequent versions of the manuscript. $\mathrm{MvH}, \mathrm{FH}, \mathrm{LPHL}$, and DvdV gave clinical advice in interpreting the results. All authors contributed critically to subsequent revisions and approved the final manuscript. The corresponding author attests that all listed authors meet authorship criteria and that no others meeting the criteria have been omitted. YO is the guarantor.

Funding: None.

Competing interests: All authors have completed the ICMJE uniform disclosure form at www.icmje.org/coi_disclosure.pdf (available on request from the corresponding author) and declare: no support from any organisation for the submitted work; no financial relationships with any organisations that might have an interest in the submitted work in the previous three years; no other relationships or activities that could appear to have influenced the submitted work.

Ethical approval: Not needed.

Data sharing: No additional data available.

Transparency: The lead author affirms that the manuscript is an honest, accurate, and transparent account of the study being reported; that no important aspects of the study have been omitted; and that any discrepancies from the study as planned (and, if relevant, registered) have been explained.

This is an Open Access article distributed in accordance with the Creative Commons Attribution Non Commercial (CC BY-NC 4.0) license, which permits others to distribute, remix, adapt, build upon this work non-commercially, and license their derivative works on different terms, provided the original work is properly cited and the use is noncommercial. See: http://creativecommons.org/licenses/by-nc/4.0/.

1 Ganestam A, Kallemose T, Troelsen A, Barfod KW. Increasing incidence of acute Achilles tendon rupture and a noticeable decline in surgical treatment from 1994 to 2013. A nationwide registry study of 33,160 patients. Knee Surg Sports Traumatol Arthrosc 2016;24:3730-7. doi:10.1007/s00167-015-3544-5

2 Egger AC, Berkowitz MJ. Achilles tendon injuries. Curr Rev Musculoskelet Med 2017:10:72-80. doi:10.1007/s12178-017-9386-7

3 Khan RJ, Carey Smith RL. Surgical interventions for treating acute Achilles tendon ruptures. Cochrane Database Syst Rev 2010;(9):CD003674.

4 Jiang N, Wang B, Chen A, Dong F, Yu B. Operative versus nonoperative treatment for acute Achilles tendon rupture: a meta-analysis based on current evidence. Int Orthop 2012;36:765-73. doi:10.1007/ s00264-011-1431-3

5 Wilkins R, Bisson LI. Operative versus nonoperative management of acute Achilles tendon ruptures: a quantitative systematic review of randomized controlled trials. Am / Sports Med 2012;40:2154-60. doi:10.1177/0363546512453293

6 Soroceanu A, Sidhwa F, Aarabi S, Kaufman A, Glazebrook M. Surgical versus nonsurgical treatment of acute Achilles tendon rupture: a meta-analysis of randomized trials. / Bone Joint Surg Am 2012:94:2136-43 doi:10.2106/BBS.K.00917

7 Erickson BJ, Mascarenhas R, Saltzman BM, et al. Is Operative Treatment of Achilles Tendon Ruptures Superior to Nonoperative Treatment?: A Systematic Review of Overlapping Metaanalyses. Orthop / Sports Med 2015:3:2325967115579188. doi:10.1177/2325967115579188

8 Benson K, Hartz AJ. A comparison of observational studies and randomized, controlled trials. N Engl / Med 2000;342:1878-86. doi:10.1056/NEJM200006223422506

9 Concato J, Shah N, Horwitz RI. Randomized, controlled trials, observational studies, and the hierarchy of research designs. N Engl/ Med 2000;342:1887-92. doi:10.1056/NEJM200006223422507

10 Anglemyer A, Horvath HT, Bero L. Healthcare outcomes assessed with observational study designs compared with those assessed in randomized trials. Cochrane Database Syst Rev 2014:(4):MR000034.

11 Abraham NS, Byrne CJ, Young JM, Solomon MJ. Meta-analysis of well-designed nonrandomized comparative studies of surgical procedures is as good as randomized controlled trials. / Clin Epidemiol 2010;63:238-45. doi:10.1016/j.jclinepi.2009.04.005

12 Arditi C, Burnand B, Peytremann-Bridevaux I. Adding non-randomised studies to a Cochrane review brings complementary information for healthcare stakeholders: an augmented systematic review and meta-analysis. BMC Health Serv Res 2016;16:598 doi:10.1186/s12913-016-1816-5

13 Frieden TR. Evidence for Health Decision Making - Beyond Randomized, Controlled Trials. N Engl J Med 2017;377:465-75. doi:10.1056/NEJMra1614394
14 Houwert RM, Smeeing DP, Ahmed Ali U, Hietbrink F, Kruyt MC, van der Meiiden OA. Plate fixation or intramedullary fixation for midshaft clavicle fractures: a systematic review and meta-analysis of randomized controlled trials and observational studies. J Shoulder Elbow Surg 2016;25:1195-203. doi:10.1016/j.je.2016.01.018

15 Smeeing DPJ, van der Ven DJC, Hietbrink F, et al. Surgical Versus Nonsurgical Treatment for Midshaft Clavicle Fractures in Patients Aged 16 Years and Older: A Systematic Review, Metaanalysis, and Comparison of Randomized Controlled Trials and Observational Studies. Am J Sports Med 2017;45:1937-45. doi:10.1177/0363546516673615

16 Beks RB, Ochen Y, Frima H, et al. Operative versus nonoperative treatment of proximal humeral fractures: a systematic review, metaanalysis, and comparison of observational studies and randomized controlled trials. J Shoulder Elbow Surg 2018;27:1526-34. doi:10.1016/i.jse.2018.03.009

17 Beks RB, Peek J, de Jong MB, et al. Fixation of flail chest or multiple rib fractures: current evidence and how to proceed. A systematic review and meta-analysis. Eur/ Trauma Emerg Surg 2018. doi:10.1007/s00068-018-1020-x

18 Stroup DF, Berlin JA, Morton SC, et al. Meta-analysis of observational studies in epidemiology: a proposal for reporting. Meta-analysis Of Observational Studies in Epidemiology (MOOSE) group. JAMA 2000;283:2008-12. doi:10.1001/jama.283.15.2008

19 Moher D, Liberati A, Tetzlaff J, Altman DG, PRISMA Group. Preferred reporting items for systematic reviews and meta-analyses: the PRISMA statement. J Clin Epidemiol 2009;62:1006-12. doi:10.1016/j.jclinepi.2009.06.005

20 Liberati A Altman DG, Tetzlaff] et al The PRISMA statement for reporting systematic reviews and meta-analyses of studies that evaluate health care interventions: explanation and elaboration. J Clin Epidemiol 2009;62:e1-34. doi:10.1016/j.jclinepi.2009.06.006

21 Slim K, Nini E, Forestier D, Kwiatkowski F, Panis Y, Chipponi J. Methodological index for non-randomized studies (minors) development and validation of a new instrument. ANZ J Surg 2003;73:712-6. doi:10.1046/j.1445-2197.2003.02748.x

22 Nilsson-Helander K, Thomeé R, Silbernagel KG, et al. The Achilles tendon Total Rupture Score (ATRS): development and validation. Am f Sports Med 2007:35:421-6. doi:10.1177/0363546506294856

23 Higgins J, Green S. Cochrane Handbook for Systematic Reviews of Interventions Version 5.1.0 (updated March 2011). 2011. http://www.cochrane.org/training/cochrane-handbook.

24 Egger M, Davey Smith G, Schneider M, Minder C. Bias in metaanalysis detected by a simple, graphical test. BMJ 1997;315:629-34. doi:10.1136/bmj.315.7109.629

25 Review Manager (RevMan) [Computer program]. Version 5.3. The Nordic Cochrane Centre, The Cochrane Collaboration, 2014

26 Lill H, Moor C, Fecht E, et al. Achilles tendon rupture - Operative or conservative functional treatment? Aktuelle Traumatol 1996;26:95-100.

27 Häggmark T, Liedberg H, Eriksson E, Wredmark T. Calf muscle atrophy and muscle function after non-operative vs operative treatment of achilles tendon ruptures. Orthopedics 1986;9:160-4.

28 Inglis AE, Scott WN, Sculco TP, Patterson AH. Ruptures of the tendo achillis. An objective assessment of surgical and nonsurgical treatment. J Bone Joint Surg Am 1976;58:990-3. doi:10.2106/00004623-197658070-00015

29 Möller M, Lind K, Movin T, Karlsson J. Calf muscle function after Achilles tendon rupture. A prospective, randomised study comparing surgical and non-surgical treatment. Scand I Med Sci Sports 2002;12:9-16. doi:10.1034/j.1600-0838.2002.120103x

30 Olsson N, Nilsson-Helander K, Karlsson J, et al. Major functional deficits persist 2 years after acute Achilles tendon rupture. Knee Surg Sports Traumatol Arthrosc 2011:19:1385-93. doi:10.1007/s00167-011-1511-3

31 Olsson N, Karlsson J, Eriksson BI, Brorsson A, Lundberg M, Silbernagel KG. Ability to perform a single heel-rise is significantly related to patient-reported outcome after Achilles tendon rupture. Scand J Med Sci Sports 2014:24:152-8. doi:10.1111/ 1600-0838.2012.01497x

32 Westin O, Nilsson Helander K, Grävare Silbernagel K, Möller M, Kälebo P, Karlsson J. Acute Ultrasonography Investigation to Predict Reruptures and Outcomes in Patients With an Achilles Tendon Rupture. Orthop / Sports Med 2016:4:2325967116667920. doi:10.1177/2325967116667920

33 Costa ML, MacMillan K, Halliday D, et al. Randomised controlled trials of immediate weight-bearing mobilisation for rupture of the tendo Achillis. J Bone Joint Surg Br 2006;88:69-77. doi:10.1302/0301-620X.88B1.16549

34 Jackson G, Sinclair VF, McLaughlin C, Barrie J. Outcomes of functional weight-bearing rehabilitation of Achilles tendon ruptures. Orthopedics 2013;36:e1053-9. doi:10.3928/0147744720130724-23

35 Kotnis R, David S, Handley R, Willett K, Ostlere S. Dynamic ultrasound as a selection tool for reducing achilles 
tendon reruptures. Am J Sports Med 2006;34:1395-400 doi:10.1177/0363546506288678

36 Bergkvist D, Åström I, Josefsson P-O, Dahlberg LE. Acute Achilles tendon rupture: a questionnaire follow-up of 487 patients. J Bone Joint Surg Am 2012;94:1229-33. doi:10.2106/JBJS.J.01601

37 Carden DG, Noble J, Chalmers J, Lunn P, Ellis J. Rupture of the calcaneal tendon. The early and late management. J Bone Joint Surg Br 1987;69:416-20. doi:10.1302/0301-620X.69B3.3294839

38 Cukelj F, Bandalovic A, Knezevic I, Pavic A, Pivalica B, Bakota B. Treatment of ruptured Achilles tendon: Operative or non-operative procedure? Injury 2015;46(Suppl 6):S137-42. doi:10.1016/j. injury.2015.10.070

39 Ebinesan AD, Sarai BS, Walley GD, Maffulli N. Conservative, open or percutaneous repair for acute rupture of the Achilles tendon. Disabil Rehabil 2008;30:1721-5. doi:10.1080/09638280701786815

40 Fahlström M, Björnstig U, Lorentzon R. Acute Achilles tendon rupture in badminton players. Am / Sports Med 1998;26:467-70. doi:10.11 77/03635465980260032201

41 Grubor P, Grubor M. Treatment of Achilles tendon rupture using different methods. Vojnosanit Pregl 2012;69:663-8. doi:10.2298/VSP1208663G

42 Gwynne-Jones DP, Sims M, Handcock D. Epidemiology and outcomes of acute Achilles tendon rupture with operative or nonoperative treatment using an identical functional bracing protocol. Foot Ankle Int 2011;32:337-43. doi:10.3113/FAl.2011.0337

43 Jaakkola JI, Beskin JL, Griffith LH, Cernansky G. Early ankle motion after triple bundle technique repair vs. casting for acute Achilles tendon rupture. Foot Ankle Int 2001;22:979-84. doi:10.1177/107110070102201210

44 Lim CS, Lees D, Gwynne-Jones DP. Functional Outcome of Acute Achilles Tendon Rupture With and Without Operative Treatment Using Identical Functional Bracing Protocol. Foot Ankle Int 2017;38:13316. doi:10.1177/1071100717728687

45 Miller D, Waterston S, Reaper J, Barrass V, Maffulli N. Conservative management, percutaneous or open repair of acute Achilles tendon rupture: a retrospective study. Scott Med I 2005;50:160-5. doi: $10.1177 / 003693300505000408$

46 Nestorson J, Movin T, Möller M, Karlsson J. Function after Achilles tendon rupture in the elderly: 25 patients older than 65 years followed for 3 years. Acta Orthop Scand 2000;71:64-8. doi:10.1080/00016470052943928

47 Rajasekar K, Gholve P, Faraj AA, Kosygan KP. A subjective outcome analysis of tendo-Achilles rupture. J Foot Ankle Surg 2005;44:32-6. doi:10.1053/j.jfas.2004.11.008

48 Renninger CH, Kuhn K, Fellars T, Youngblood S, Bellamy J. Operative and Nonoperative Management of Achilles Tendon Ruptures in Active Duty Military Population. Foot Ankle Int 2016;37:269-73. doi:10.1177/1071100715615322

49 van der Linden-van der Zwaag HMJ, Nelissen RGHH, Sintenie JB. Results of surgical versus non-surgical treatment of Achilles tendon rupture. Int Orthop 2004;28:370-3. doi:10.1007/s00264-004-0575-9

50 Wang D, Sandlin MI, Cohen JR, Lord EL, Petrigliano FA, SooHoo NF. Operative versus nonoperative treatment of acute Achilles tendon rupture: An analysis of 12,570 patients in a large healthcare database. Foot Ankle Surg 2015;21:250-3. doi:10.1016/j.fas.2015.01.009

51 Weber M, Niemann M, Lanz R, Müller T. Nonoperative treatment of acute rupture of the achilles tendon: results of a new protocol and comparison with operative treatment. Am J Sports Med 2003;31:685-91. doi:10.1177/03635465030310050901

52 Cetti R, Christensen SE, Ejsted R, Jensen NM, Jorgensen U. Operative versus nonoperative treatment of Achilles tendon rupture. A prospective randomized study and review of the literature. Am J Sports Med 1993;21:791-9. doi:10.1177/036354659302100606

53 Keating JF, Will EM. Operative versus non-operative treatment of acute rupture of tendo Achillis: a prospective randomised evaluation of functional outcome. J Bone Joint Surg Br 2011;93:1071-8. doi:10.1302/0301-620X.93B8.25998

54 Lantto I, Heikkinen J, Flinkkila T, et al. A Prospective Randomized Trial Comparing Surgical and Nonsurgical Treatments of Acute Achilles Tendon Ruptures. Am J Sports Med 2016;44:2406-14. doi:10.1177/0363546516651060

55 Metz R, Verleisdonk EJ, van der Heijden GJ, et al. Acute Achilles tendon rupture: minimally invasive surgery versus nonoperative treatment with immediate full weightbearing--a randomized controlled trial. Am J Sports Med 2008;36:1688-94 doi:10.1177/0363546508319312

56 Möller M, Movin T, Granhed H, Lind K, Faxén E, Karlsson J. Acute rupture of tendon Achillis. A prospective randomised study of comparison between surgical and non-surgical treatment. I Bone Joint Surg Br 2001;83:843-8. doi:10.1302/0301 620X.83B6.0830843

57 Nistor L. Surgical and non-surgical treatment of Achilles Tendon rupture. A prospective randomized study. J Bone Joint Surg Am 1981;63:394-9. doi:10.2106/00004623198163030-00012

58 Nilsson-Helander K, Silbernagel KG, Thomeé R, et al. Acute achilles tendon rupture: a randomized, controlled study comparing surgical and nonsurgical treatments using validated outcome measures. Am / Sports Med 2010;38:2186-93. doi:10.1177/0363546510376052

59 Olsson N, Silbernagel KG, Eriksson BI, et al. Stable surgica repair with accelerated rehabilitation versus nonsurgical treatment for acute Achilles tendon ruptures: a randomized controlled study. Am J Sports Med 2013;41:2867-76. doi:10.1177/0363546513503282

60 Twaddle BC, Poon P. Early motion for Achilles tendon ruptures: is surgery important? A randomized, prospective study. Am J Sports Med 2007;35:2033-8. doi:10.1177/0363546507307503

61 Willits K, Amendola A, Bryant D, et al. Operative versus nonoperative treatment of acute Achilles tendon ruptures: a multicenter randomized trial using accelerated functional rehabilitation. J Bone Joint Surg Am 2010;92:2767-75. doi:10.2106/JBJS.I.01401

62 Majewski M, Rickert M, Steinbrück K. [Achilles tendon rupture. A prospective study assessing various treatment possibilities] Orthopade 2000;29:670-6.

63 van der Eng DM, Schepers T, Goslings JC, Schep NW. Rerupture rate after early weightbearing in operative versus conservative treatment of Achilles tendon ruptures: a meta-analysis. J Foot Ankle Surg 2013;52:622-8. doi:10.1053/j.jfas.2013.03.027

64 De Boer AS, Van Lieshout EMM, Van Moolenbroek G, Den Hartog D, Verhofstad MHJ. The effect of time to post-operative weightbearing on functional and clinical outcomes in adults with a displaced intraarticular calcaneal fracture; A systematic review and pooled analysis. Injury 2018;49:743-52. doi:10.1016/j.injury.2018.02.021

65 Smeeing DPJ, Houwert RM, Briet JP, et al. Weight-bearing and mobilization in the postoperative care of ankle fractures: a systematic review and meta-analysis of randomized controlled trials and cohort studies. PLoS One 2015;10:e0118320. doi:10.1371/journal.pone.0118320

66 Eliasson P, Agergaard A-S, Couppé C, et al. The Ruptured Achilles Tendon Elongates for 6 Months After Surgical Repair Regardless of Early or Late Weightbearing in Combination With Ankle Mobilization: A Randomized Clinical Trial. Am J Sports Med 2018;46:2492-502. doi:10.1177/0363546518781826

67 Yang B, Liu Y, Kan S, et al. Outcomes and complications of percutaneous versus open repair of acute Achilles tendon rupture: A meta-analysis. Int J Surg 2017;40:178-86. doi:10.1016/j. ijsu.2017.03.021

68 loannidis JP, Haidich AB, Pappa M, et al. Comparison of evidence of treatment effects in randomized and nonrandomized studies. JAMA 2001;286:821-30. doi:10.1001/jama.286.7.821

69 Hemkens LG, Contopoulos-Ioannidis DG, loannidis JPA. Agreement of treatment effects for mortality from routinely collected data and subsequent randomized trials: meta-epidemiological survey. BMJ 2016;352:i493. doi:10.1136/bmj.i493

70 Boyko EJ. Observational research--opportunities and limitations. Diabetes Complications 2013;27:642-8. doi:10.1016/j. jdiacomp.2013.07.007

71 Van Spall HGC, Toren A, Kiss A, Fowler RA. Eligibility criteria of randomized controlled trials published in high-impact general medical journals: a systematic sampling review. JAMA 2007;297:1233-40. doi:10.1001/jama.297.11.1233

72 Khan AY, Preskorn SH, Baker B. Effect of study criteria on recruitment and generalizability of the results. J Clin Psychopharmacol 2005;25:271-5. doi:10.1097/01. jcp.0000161497.73514.80

\section{Supplementary materials}

